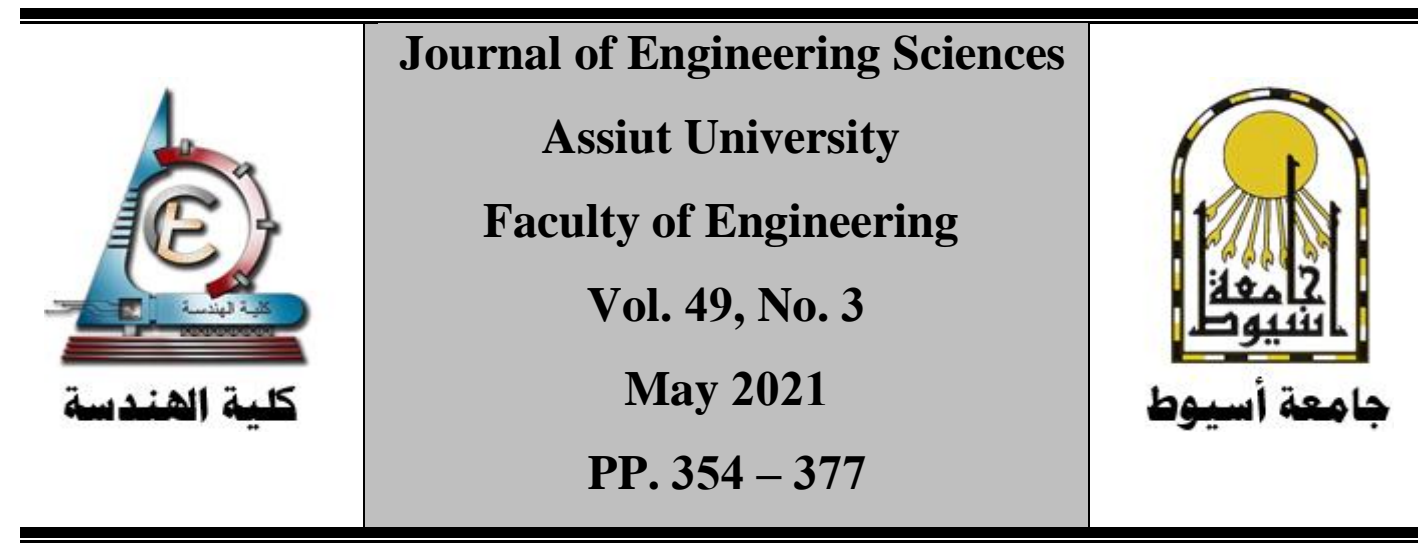

\title{
A NEW APPROACH FOR WATER ALLOCATION SCHEDULING IN IRRIGATION OPEN CHANNELS CASE STUDY: ALMANNA MAIN CANAL, AND ITS BRANCHES
}

Tarek Sayed Abuzeid ${ }^{1}$ and Mahmoud Sabery ${ }^{2}$

\author{
${ }^{l}$ Lecturer, Civil Engineering Department, Assiut University, Assiut, Egypt \\ *tareksayed1986@aun.edu.eg \\ ${ }^{2}$ Ph.D. Researcher, Ministry of Water Resources and Irrigation, Egypt
}

Received 7 March 2021; Revised 04 May 2021; Accepted 04 May 2021

\begin{abstract}
In Egypt, the Agriculture sector consumes about $85.90 \%$ of the total actual consumption of water. The irrigation canals network in Egypt at all levels suffer from water losses, distorted sections, and un-equitable distribution of irrigation water among beneficiaries. With population growth, expansion of economic and industrial activities and entry of Egypt into the stage of water poverty, rationalization of the consumption of irrigation water has become an urgent requirement and a key factor in achieving sustainable water development. Although crops may need different water quantities during their growth stages, irrigation canals are constantly supplied with almost the same monthly discharge during periods of peak or minimum requirements. The objective of this study is to activate a new approach for the reasonable management of the irrigation network for the study area. This approach allows the canals network to be supplied with monthly discharges consistent with the actual water consumption of the cultivated plant. Also, it helps in rationalizing consumption during the agricultural season. The new approach was applied to Almanna main canal and its branches which belong to the Assiut governorate in Egypt, as an example of the irrigation system in Egypt. Rotational distribution is practiced at the distributary canal level. The results
\end{abstract}


indicated that applying the new approach on Almanna distributary canals can save a large amount of irrigation water that reaches about 19.375 Million cubic meters monthly, representing $48.68 \%$ of all irrigation water given to Almanna distributary canals. Also, the percentage value of the new discharges of Almanna distributary canals, which calculated according to the water needs of crops cultivated in the area, ranged between 32 and $67 \%$ of the designed discharges.

Keywords: Water Consumption; Crop Water Requirements; Water Allocation.

\section{Introduction}

The growing demand for water has ushered in the need for effective use of water in the irrigation sector with different methods of management. The main aim of these methods is to meet the crop demand with the available water to get maximum production. Scheduling of water delivery is one among them and it is a core activity that has more influence on the performance of the system compared to other irrigation activities [1]. Scheduling of an irrigation water distribution system should be based on the objectives or targets of the irrigation system that can be measured with performance indicators. The concepts and definitions of performance indicators that describe the quality of irrigation service provided by the managers of the water delivery system have been presented by many researchers such as given by $[\mathbf{2}, \mathbf{3}, \mathbf{4}, \mathbf{5}, \mathbf{6} \boldsymbol{\&} \mathbf{7}]$. It is also realized that there are complexities in identifying the objectives, defining them, and assessing them at different levels of an irrigation system by different interest groups with differing perspectives. The complexities are well described in the literature [1\& 8].

Today, equity, adequacy, and timeliness are the main objectives of canal water distribution procedures. These operational objectives may be conflicting with or contributing to each other in different magnitudes depending upon the water availability [9]. The canal was designed as continuous water supply systems. The increase in cropping area and changes in cropping pattern in course of time increase the demand in these systems. So, the main canal capacity is inadequate to run all the distributary canals simultaneously. Rotational water 
distribution has been introduced in some of the systems to manage the shortage of water.

In most of the irrigation systems, the manager who prepares the irrigation schedule relies mainly on his experience or rules of thumb. He distributes the water to the canals according to the pre-specified water duty. This water duty does not vary with time. It gives an approximate estimation of water required for a virtual cropping pattern over a gross period like a season. If the canals are large in number and vary in design discharge, length and served area, the manager requires special skill to set water allocating priorities for the defined objectives, develop and implement an irrigation schedule under these complex situations. Several models have been developed for irrigation scheduling with optimization and simulation techniques $[10,11$, $12 \& 13]$. A procedure for the operation of canals using a water balance equation have been developed by Rajput and Michael [14], for the estimation of daily soil moisture status taking a hypothetical case of four branch canal system. This model can be applied to real situations only if the number of branch canals in the network is in multiples of four. Vedula et al. [15] used dynamic programming to develop an irrigation scheduling model for optimal allocation of water during different periods of the season for a single crop. The model considered the soil moisture contribution for estimating the irrigation requirement. Also, Kalu et al. [16] used compromise programming to select the best water distribution policies according to crop yield, efficiency, and equity measures. The model was used in a small command area and emphasis was put on crop production in relation to the water supply. An adjusted irrigation plan proposed for water distribution in large areas by Kan and Shu [17] through lengthening the rotational irrigation periods in no water shortage conditions. This plan allowed good water distribution but it was much limited when under large stresses water supplies considerably decreased. Water distribution hence became a bottleneck for both water supply system and farmers. Farmers are under pressure to grow more, so there is an urgent need to find ways to grow more with less water [18]. A multi-criteria mathematical model for canal irrigation scheduling in rotational distribution was formulated to achieve the objectives of minimizing gate operations, improving the performance of equity, adequacy, and timeliness [19]. Yuanhua and Honyuan [20] evolved a model for canal scheduling with rotational water distribution by estimating the initial soil moisture daily through 
the water balance equation and forecasting the weather data and subsequently the irrigation date and depth. Similar observations are discussed by Hill and Allen [21] while developing an irrigation scheduling calendar in Pakistan. Also, the application of the $0 \pm 1$ linear programming model proposed by Zhi et al. [22] for outlet schedule is limited to irrigation systems where the distribution outlets along the canal (be it main, lateral, tertiary) have the same discharge capacity and such systems are hypothetical. Most of these models have difficulties in field applications for the following reasons: (a) the assumptions made and or the pre-defined mathematical structure involved in developing the optimization problem do not match with the real conditions of the field, and (b) the field measurement data required for these models such as the soil moisture status or plant stress are generally not collected and used in most of the irrigation systems in many countries.

Tests to reduce the non-flexibility in matching irrigation intervals and crop or soil condition are being carried out in Egypt but are hampered by the fact that the cropping pattern is not known, and water flows/volumes cannot be properly measured and regulated [23]. In practice, this means that hopes for a better match between irrigation supplies and demand remain futile until a more accurate regulation of the available volumes is possible, provided the water is available. Rather than the lack of awareness about the need to develop the capacity to deal with the probable future droughts still exists.

Irrigation canals network in Egypt at all levels suffer from water losses, distorted sections, and un-equitable distribution of irrigation water among beneficiaries. With population growth, expansion of economic and industrial activities and entry of Egypt into the stage of water poverty, rationalization of the consumption of irrigation water has become an urgent requirement and a key factor in achieving sustainable water development. Although crops may need different water quantities during their growth stages, irrigation canals are constantly supplied with almost the same monthly discharge during periods of peak or minimum requirements. The objective of this study is to activate a new approach for the reasonable management of the irrigation network for the study area. This approach allows the canals network to be supplied with monthly discharges consistent with the actual water consumption of the cultivated plant. Also, it helps in rationalizing consumption during the agricultural season. The new 
approach was applied to Almanna main canal and its branches which belong to the Assiut governorate in Egypt, as an example of the irrigation system in Egypt. Rotational distribution is practiced at the distributary canal level.

\section{Materials and Methods}

\subsection{Description of the study area}

Almanna canal was chosen to conduct the present field study as a representative open channel having specific properties from different technical points of view, soil type, weather condition, and the length with its off-taking canals. Almanna canal belongs to Abnoub Irrigation Engineering Administration in Assiut. The length of Almanna canal is about $32.80 \mathrm{~km}$ and its intake located at $\mathrm{km} 157$ at the right bank for the Eastern Naga Hammady canal as shown in Fig. (1). Twenty distributary canals are branched from Almanna canal on both sides as shown in Fig. (2). Total length of the branches is about $47.097 \mathrm{~km}$. Table (1) shows geometrical characteristics and discharges of the different sections of Almanna canal. It was firstly constructed between Al-Matmar and Al-Maabda to carry fresh water for municipal water supply. It has four regulators constructed along its total length. The first one is a head regulator at $\mathrm{km}(0.000)$, the second regulator at $\mathrm{km}$. (10.450), the third one at $\mathrm{km} .(16.450)$, and the fourth regulator at $\mathrm{km}$. (23.060). Table (2) presents the geometric and hydraulic characteristics of Almanna off-taking canals that can be used to assess and propose the water management criteria. Manning equation is used for calculating the designed discharges of canals as given in Tables (1), and (2). Manning equation in the metric system could be written as:

$$
Q=\frac{1}{n} \times S^{0.5} \times A \times R^{2 / 3}
$$

where Q: water discharge $\left(\mathrm{m}^{3} / \mathrm{s}\right)$, S: friction slope could be substituted by water slope as demonstrated in Tables (1 and 2) $(\mathrm{m} / \mathrm{m}), \mathrm{R}$ : hydraulic radius $(\mathrm{m})$, and $\mathrm{A}$ : area of hydraulic section $\left(\mathrm{m}^{2}\right)$.

The study area is located between $27^{\circ} 16^{\prime}$ and $27^{\circ} 33^{\prime}$ north Latitude and between $31^{\circ} 27^{\prime}$ and $31^{\circ} 01^{\prime}$ east Longitude. The area is characterized by arid climate. Tables (3 and 4) show statistic models for the 
meteorological data of Abnoub cropped area during a period of 5 years started from 2014 to 2019 as documented in the Arab Alawlamer weather station [24]. The agricultural land is at 50 meters above the mean sea water level. Canal cropped area is 13500 feddans. Winter crops are Wheat, Clover, Bean, while summer ones are Yellow corn, Sorghum, and Basil. Table (5) shows the areas of different crops cultivated during the last 5 years. This table was taken from the Agriculture Directorate in Assiut Governorate. It is noticed from this table that, the areas of cultivated lands differ during the five years, and this may be due to economic, social, or agricultural reasons of the beneficiaries that require the un-constant of the cultivated area. Consequently, there are wastelands that have not been cultivated, so they were not considered when calculating the actual cultivated area. These data were used to calculate the percentage of cultivation of these crops. Through these ratios, the area of cultivation of each crop was calculated as shown in Table (6). By knowing the water requirements of each crop, it is possible to predict the optimum discharge of all canals in the study area.

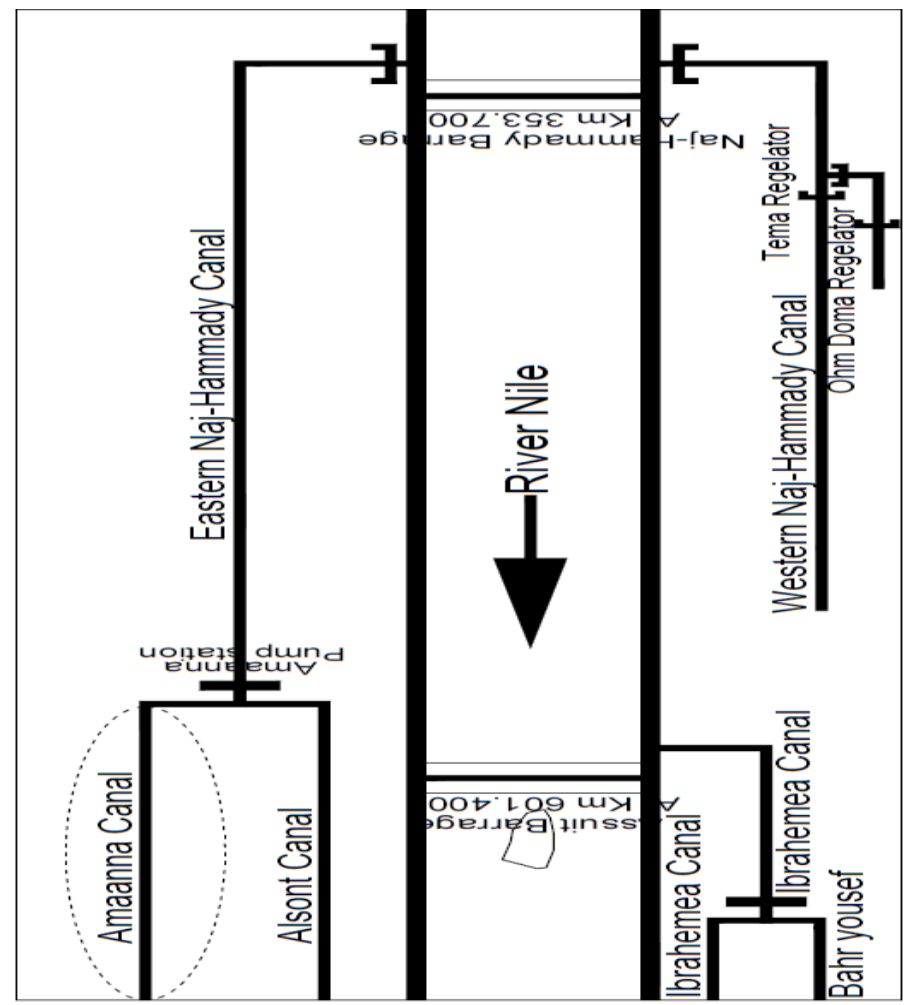

Fig. 1. Synoptic of regional canals' network 


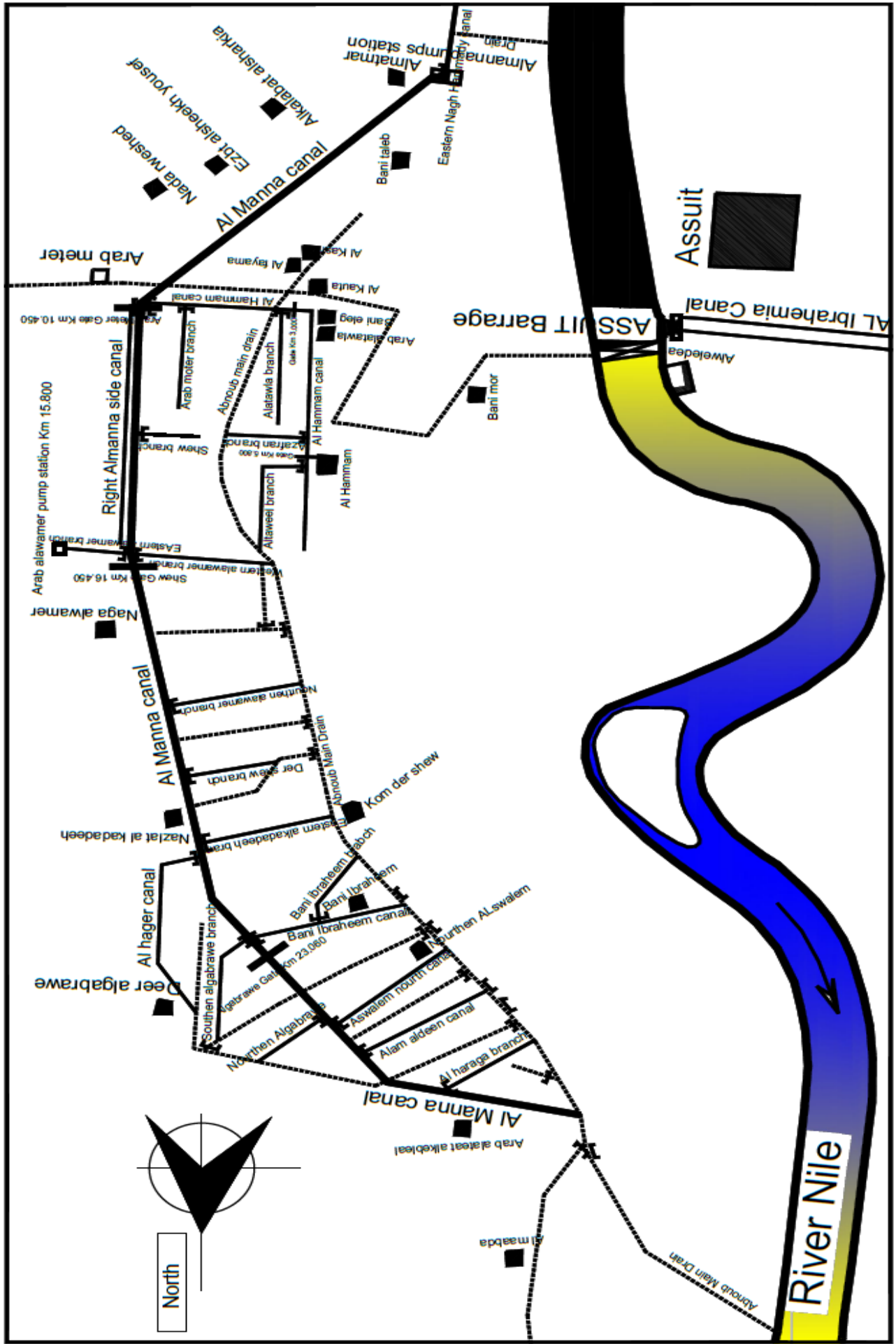

Fig. 2. Layout of Al-Manna canal and its branches 
Tarek S, Abuzeid, A New Approach for Water Allocation Scheduling in Irrigation Open Channels......

Table 1. Geometrical characteristics and discharges of the different section of Almanna canal.

\begin{tabular}{|c|c|c|c|c|c|c|c|c|c|c|}
\hline No & Sections $(\mathrm{Km})$ & Gate & $\begin{array}{c}\text { Area } \\
\text { (Fedd) }\end{array}$ & $\begin{array}{c}\text { Length } \\
(\mathrm{Km})\end{array}$ & $\begin{array}{l}\text { Side } \\
\text { slope }\end{array}$ & $\begin{array}{c}\text { longitudinal } \\
\text { water slope (-) }\end{array}$ & $\begin{array}{l}\text { Bed width } \\
\text { (m) }\end{array}$ & \multicolumn{2}{|c|}{ Levels (m) } & $\begin{array}{l}\mathrm{Q} \text { Designe } \\
\left(\mathrm{m}^{3} / \mathrm{sec}\right)\end{array}$ \\
\hline 2 & $(10.45-16.45)$ & Arab Meter-Shew & 7450 & 6.0000 & $3: 2$ & 0.00005 & 7.50 & 48.30 & 51.00 & 18.55 \\
\hline 3 & $(16.45-23.06)$ & Shew- Algabrawe & 3280 & 6.6100 & $3: 2$ & 0.00005 & 6.00 & 48.00 & 50.40 & 14.65 \\
\hline 4 & $(23.06-26.40)$ & Algabrawe - End & 2600 & 3.3400 & $3: 2$ & 0.00010 & 4.00 & 47.67 & 49.73 & 8.23 \\
\hline 5 & $(26.40-32.80)$ & End & 170.0 & 6.4000 & $3: 2$ & 0.00005 & 3.00 & 47.25 & 49.42 & 7.55 \\
\hline
\end{tabular}

Table 2. Geometrical characteristics and discharges of Almanna off-taking canals

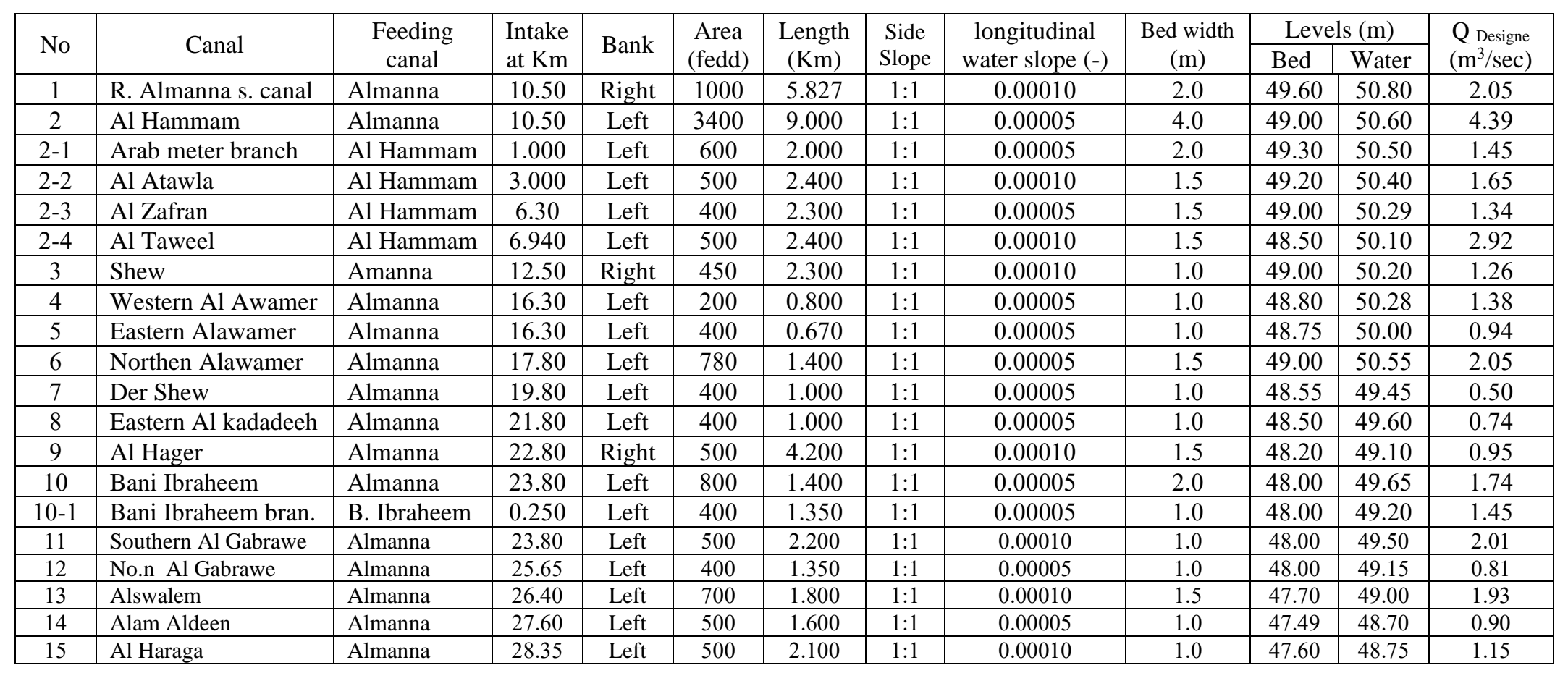


Table 3. Average monthly meteorological data of Abnoub area during winter growing seasons [24].

\begin{tabular}{|c|c|c|c|c|c|c|c|}
\hline \multicolumn{8}{|c|}{ Season of $2013 / 2014$} \\
\hline \multirow{2}{*}{ Month } & \multicolumn{2}{|c|}{$\begin{array}{c}\text { Temperature } \\
\left(\mathrm{C}^{\circ}\right)\end{array}$} & \multirow{2}{*}{$\begin{array}{c}\text { Relative } \\
\text { humidity } \\
\%\end{array}$} & \multirow{2}{*}{$\begin{array}{c}\text { E pan } \\
\text { mm/day }\end{array}$} & \multirow{2}{*}{$\begin{array}{l}\text { Wind } \\
\text { Speed } \\
\mathrm{Km} / \mathrm{h} \\
\end{array}$} & \multirow{2}{*}{$\begin{array}{c}\text { Solar } \\
\text { radiation } \\
\mathrm{MJ} / \mathrm{m} 2 / \text { day }\end{array}$} & \multirow{2}{*}{$\begin{array}{c}\text { No. of } \\
\text { sunny } \\
\text { hours / day }\end{array}$} \\
\hline & $\operatorname{Max}$ & Min & & & & & \\
\hline December & 23.2 & 8.50 & 48.40 & 5.67 & 14.40 & 14.80 & 9.00 \\
\hline January & 22.4 & 6.30 & 55.20 & 2.82 & 07.90 & 15.40 & 8.90 \\
\hline February & 23.8 & 7.40 & 49.20 & 3.15 & 12.00 & 18.70 & 9.70 \\
\hline March & 27.9 & 12.1 & 42.40 & 4.43 & 11.50 & 21.70 & 9.90 \\
\hline April & 32.2 & 15.8 & 34.90 & 6.41 & 10.92 & 24.50 & 10.3 \\
\hline \multicolumn{8}{|c|}{ Season of $2014 / 2015$} \\
\hline December & 20.4 & 7.20 & 63.20 & 3.11 & 16.80 & 14.80 & 9.0 \\
\hline January & 20.5 & 5.50 & 44.00 & 2.32 & 16.00 & 15.40 & 8.9 \\
\hline February & 22.7 & 7.60 & 38.80 & 3.08 & 16.40 & 18.70 & 9.7 \\
\hline March & 27.2 & 12.2 & 34.00 & 3.79 & 19.40 & 21.70 & 9.9 \\
\hline April & 29.3 & 14.6 & 25.60 & 6.33 & 19.90 & 24.50 & 10.3 \\
\hline \multicolumn{8}{|c|}{ Season of $2015 / 2016$} \\
\hline December & 19.50 & 6.30 & 59.70 & 2.50 & 16.80 & 14.80 & 9.0 \\
\hline January & 19.00 & 5.10 & 60.30 & 2.17 & 15.20 & 15.40 & 8.9 \\
\hline February & 24.50 & 8.30 & 50.70 & 2.74 & 14.50 & 18.70 & 9.7 \\
\hline March & 28.00 & 13.10 & 41.00 & 3.99 & 17.00 & 21.70 & 9.9 \\
\hline April & 35.10 & 17.10 & 31.50 & 6.40 & 17.00 & 24.50 & 10.3 \\
\hline \multicolumn{8}{|c|}{ Season of 2016/2017 } \\
\hline December & 23.20 & 9.00 & 58.80 & 5.67 & 14.60 & 14.80 & 9.00 \\
\hline January & 19.30 & 5.30 & 55.30 & 2.82 & 14.80 & 15.40 & 8.90 \\
\hline February & 20.50 & 6.30 & 52.60 & 3.15 & 14.50 & 18.70 & 9.70 \\
\hline March & 25.30 & 11.00 & 42.50 & 4.43 & 17.20 & 21.70 & 9.90 \\
\hline April & 31.30 & 15.50 & 36.60 & 6.41 & 17.30 & 24.50 & 10.3 \\
\hline \multicolumn{8}{|c|}{ Season of $2017 / 2018$} \\
\hline December & 20.80 & 8.00 & 62.80 & 3.11 & 16.30 & 14.80 & 9.0 \\
\hline January & 19.90 & 6.50 & 57.50 & 2.32 & 15.30 & 15.40 & 8.9 \\
\hline February & 26.10 & 11.20 & 44.30 & 3.08 & 14.40 & 18.70 & 9.7 \\
\hline March & 30.50 & 14.20 & 36.20 & 3.79 & 16.90 & 21.70 & 9.9 \\
\hline April & 32.40 & 16.60 & 36.20 & 6.33 & 18.40 & 24.50 & 10.3 \\
\hline \multicolumn{8}{|c|}{ Season of 2019/2020 } \\
\hline December & 23.20 & 9.00 & 58.80 & 2.50 & 14.60 & 14.80 & 9.0 \\
\hline January & 19.30 & 5.80 & 52.80 & 2.18 & 13.90 & 15.40 & 8.9 \\
\hline February & 21.80 & 7.60 & 51.40 & 2.75 & 17.30 & 18.70 & 9.7 \\
\hline March & 24.70 & 9.90 & 42.90 & 4.01 & 19.80 & 21.70 & 9.9 \\
\hline April & 29.60 & 14.00 & 36.50 & 6.22 & 21.30 & 24.50 & 10.3 \\
\hline
\end{tabular}


Tarek S, Abuzeid, A New Approach for Water Allocation Scheduling in Irrigation Open Channels......

Table 4. Average monthly meteorological data of Abnoub area during summer growing seasons [24]:

\begin{tabular}{|c|c|c|c|c|c|c|c|}
\hline \multicolumn{8}{|c|}{ Season of 2014} \\
\hline \multirow[t]{2}{*}{ Month } & \multicolumn{2}{|c|}{$\begin{array}{c}\text { Temperature } \\
\left(\mathrm{C}^{\circ}\right)\end{array}$} & \multirow{2}{*}{$\begin{array}{c}\text { Relative } \\
\text { humidity } \\
\%\end{array}$} & \multirow{2}{*}{$\begin{array}{c}\text { E pan } \\
\mathrm{mm} / \text { day }\end{array}$} & \multirow{2}{*}{$\begin{array}{l}\text { Wind } \\
\text { Speed } \\
\text { Km/h }\end{array}$} & \multirow{2}{*}{$\begin{array}{c}\text { Solar } \\
\text { radiation } \\
\mathrm{MJ} / \mathrm{m}^{2} / \text { day }\end{array}$} & \multirow{2}{*}{$\begin{array}{c}\text { No. of } \\
\text { sunny } \\
\text { hours / day }\end{array}$} \\
\hline & Min & Max & & & & & \\
\hline May & 35.60 & 19.70 & 35.10 & 6.52 & 10.70 & 27.00 & 11.40 \\
\hline June & 37.80 & 22.30 & 33.20 & 6.61 & 12.40 & 28.50 & 12.30 \\
\hline July & 38.30 & 23.60 & 32.00 & 6.70 & 12.80 & 28.20 & 12.20 \\
\hline August & 38.40 & 23.90 & 33.80 & 7.10 & 14.40 & 27.10 & 11.90 \\
\hline September & 35.80 & 22.10 & 33.60 & 5.00 & 15.80 & 23.70 & 10.80 \\
\hline October & 31.30 & 16.90 & 36.70 & 4.60 & 9.80 & 19.90 & 10.00 \\
\hline November & 26.60 & 12.30 & 45.00 & 4.45 & 11.80 & 16.50 & 9.40 \\
\hline \multicolumn{8}{|c|}{ Season of 2015} \\
\hline May & 35.50 & 19.70 & 27.10 & 6.34 & 17.40 & 27.00 & 11.40 \\
\hline June & 36.60 & 21.30 & 37.40 & 6.39 & 13.00 & 28.50 & 12.30 \\
\hline July & 38.80 & 22.80 & 35.90 & 6.78 & 8.20 & 28.20 & 12.20 \\
\hline August & 40.30 & 24.80 & 38.60 & 7.44 & 8.40 & 27.10 & 11.90 \\
\hline September & 38.50 & 23.80 & 38.50 & 5.89 & 14.60 & 23.70 & 10.80 \\
\hline October & 33.00 & 19.50 & 51.30 & 4.89 & 16.30 & 19.90 & 10.00 \\
\hline November & 26.30 & 13.20 & 59.50 & 4.51 & 15.20 & 16.50 & 9.40 \\
\hline \multicolumn{8}{|c|}{ Season of 2016} \\
\hline May & 36.10 & 20.00 & 27.70 & 6.48 & 20.30 & 27.00 & 11.40 \\
\hline June & 40.70 & 24.60 & 28.00 & 6.53 & 19.50 & 28.50 & 12.30 \\
\hline July & 37.40 & 24.10 & 37.90 & 7.20 & 19.50 & 28.20 & 12.20 \\
\hline August & 37.50 & 24.10 & 36.80 & 7.10 & 19.50 & 27.10 & 11.90 \\
\hline September & 35.00 & 21.60 & 43.50 & 7.01 & 21.70 & 23.70 & 10.80 \\
\hline October & 32.80 & 17.70 & 49.50 & 5.60 & 19.20 & 19.90 & 10.00 \\
\hline November & 27.00 & 12.70 & 54.70 & 4.62 & 15.10 & 16.50 & 9.40 \\
\hline \multicolumn{8}{|c|}{ Season of 2017} \\
\hline May & 36.30 & 20.00 & 31.40 & 6.56 & 16.20 & 27.00 & 11.40 \\
\hline June & 37.40 & 23.40 & 34.60 & 6.67 & 21.00 & 28.50 & 12.30 \\
\hline July & 39.10 & 25.30 & 32.70 & 6.76 & 16.30 & 28.20 & 12.20 \\
\hline August & 37.80 & 24.60 & 38.80 & 7.42 & 17.60 & 27.10 & 11.90 \\
\hline September & 35.30 & 20.90 & 44.60 & 5.87 & 20.70 & 23.70 & 10.80 \\
\hline October & 30.30 & 16.50 & 47.00 & 4.86 & 17.20 & 19.90 & 10.00 \\
\hline November & 25.10 & 10.90 & 54.60 & 4.49 & 15.20 & 16.50 & 9.40 \\
\hline \multicolumn{8}{|c|}{ Season of 2018} \\
\hline May & 37.70 & 21.70 & 29.20 & 6.38 & 17.50 & 27.00 & 11.40 \\
\hline June & 38.50 & 23.20 & 33.60 & 6.68 & 20.00 & 28.50 & 12.30 \\
\hline July & 38.00 & 24.70 & 41.50 & 7.05 & 18.70 & 28.20 & 12.20 \\
\hline August & 37.60 & 24.30 & 40.70 & 7.77 & 19.80 & 27.10 & 11.90 \\
\hline September & 35.50 & 22.00 & 46.20 & 6.02 & 20.50 & 23.70 & 10.80 \\
\hline October & 32.60 & 18.90 & 46.50 & 5.52 & 18.10 & 19.90 & 10.00 \\
\hline November & 26.50 & 13.10 & 53.80 & 4.98 & 14.70 & 16.50 & 9.40 \\
\hline
\end{tabular}


JES, Assiut University, Faculty of Engineering, Vol.49, No. 3, May 2021, pp. 354 - 377

\begin{tabular}{|l|c|c|c|c|c|c|c|}
\hline \multicolumn{7}{|l|}{ Continue...... } \\
\hline May & 38.10 & 22.00 & 28.90 & 6.56 & 18.90 & 27.00 & 11.40 \\
\hline June & 39.00 & 24.90 & 33.90 & 6.89 & 20.30 & 28.50 & 12.30 \\
\hline July & 39.10 & 25.30 & 32.70 & 7.41 & 16.30 & 28.20 & 12.20 \\
\hline August & 37.80 & 24.60 & 38.80 & 7.99 & 17.60 & 27.10 & 11.90 \\
\hline September & 35.30 & 20.90 & 44.60 & 6.45 & 20.70 & 23.70 & 10.80 \\
\hline October & 30.30 & 16.50 & 47.00 & 5.71 & 17.20 & 19.90 & 10.00 \\
\hline November & 25.10 & 10.90 & 54.60 & 4.84 & 15.20 & 16.50 & 9.40 \\
\hline
\end{tabular}

Table 5. Seasonal cropping pattern in Abnoub cropped area

\begin{tabular}{|c|c|c|c|c|c|c|c|c|c|}
\hline \multirow{2}{*}{ 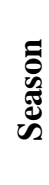 } & \multirow[b]{2}{*}{ Crop } & \multicolumn{5}{|c|}{ Area (feddan) } & \multirow[b]{2}{*}{$\stackrel{\mathscr{E}}{\Xi}$} & \multirow{2}{*}{ 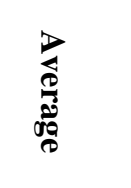 } & \multirow{2}{*}{ 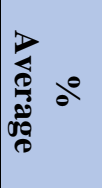 } \\
\hline & & $\begin{array}{c}2015 \\
: \\
2016\end{array}$ & $\begin{array}{c}2016 \\
: \\
2017\end{array}$ & $\begin{array}{c}2017 \\
: \\
2018\end{array}$ & $\begin{array}{c}2018 \\
: \\
2019\end{array}$ & $\begin{array}{c}2019 \\
: \\
2020\end{array}$ & & & \\
\hline \multirow{4}{*}{$\stackrel{\bar{\Xi}}{:}$} & Wheat & 23500 & 21500 & 24200 & 18900 & 28590 & 116690 & 23338 & 60.90 \\
\hline & Clover & 11750 & 11500 & 10500 & 13200 & 10100 & 57050 & 11410 & 29.80 \\
\hline & Bean & - & 1000 & 9550 & 3915 & 3425 & 17890 & 3578 & 9.30 \\
\hline & Summation & 35250 & 34000 & 44250 & 36015 & 42115 & 191630 & 38326 & \\
\hline \multirow{4}{*}{$\begin{array}{l}\dot{\Phi} \\
\stackrel{\Xi}{\Xi} \\
\vdots\end{array}$} & Y- corn & 9500 & 21000 & 20000 & 19570 & 15500 & 85570 & 17114 & 52.40 \\
\hline & Sorghum & 13975 & 8000 & 10000 & 11100 & 10700 & 53775 & 10755 & 32.90 \\
\hline & Basil & 2000 & 6250 & 5250 & 4445 & 5968 & 23913 & 4782.6 & 14.60 \\
\hline & Summation & 25475 & 35250 & 35250 & 35115 & 32168 & 163258 & 32651.6 & \\
\hline
\end{tabular}

Table 6. Areas of winter and summer crops in Almanna off-taking canals.

\begin{tabular}{|c|c|c|c|c|c|c|c|c|}
\hline \multirow[b]{2}{*}{ No } & \multirow[b]{2}{*}{ Canal } & \multirow[b]{2}{*}{$\begin{array}{c}\text { Area } \\
\text { (fedd) }\end{array}$} & \multicolumn{3}{|c|}{$\begin{array}{l}\text { Areas of winter crops } \\
\qquad\left(\mathrm{A}_{\mathrm{ci}}\right)\end{array}$} & \multicolumn{3}{|c|}{$\begin{array}{l}\text { Areas of summer crops } \\
\left(\mathrm{A}_{\mathrm{ci}}\right)\end{array}$} \\
\hline & & & 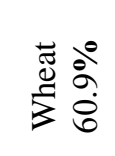 & 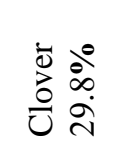 & ॠี & 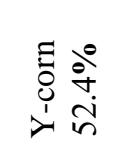 & 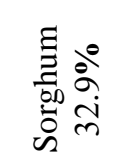 & 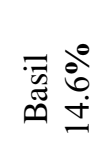 \\
\hline 1 & $\begin{array}{l}\text { Right Almanna } \\
\text { side canal }\end{array}$ & 1000 & 609 & 298 & 93 & 524 & 329 & 146 \\
\hline 2 & Al Hammam & 3400 & 2070.6 & 1013.2 & 316.2 & 1781.6 & 1118.6 & 496.4 \\
\hline $2--1$ & $\begin{array}{l}\text { Arab meter } \\
\text { branch }\end{array}$ & 600 & 365.4 & 178.8 & 55.8 & 314.4 & 197.4 & 87.6 \\
\hline $2--2$ & Al Atawla & 500 & 304.5 & 149 & 46.5 & 262 & 164.5 & 73 \\
\hline $2--3$ & Al Zafran & 400 & 243.6 & 119.2 & 37.2 & 209.6 & 131.6 & 58.4 \\
\hline $2--4$ & Al Taweel & 500 & 304.5 & 149 & 46.5 & 262 & 164.5 & 73 \\
\hline 3 & Shew & 450 & 274.05 & 134.1 & 41.85 & 235.8 & 148.05 & 65.7 \\
\hline 4 & $\begin{array}{l}\text { Western Al } \\
\text { Awamer }\end{array}$ & 200 & 121.8 & 59.6 & 18.6 & 104.8 & 65.8 & 29.2 \\
\hline 5 & Eastern & 400 & 243.6 & 119.2 & 37.2 & 209.6 & 131.6 & 58.4 \\
\hline
\end{tabular}


Tarek S, Abuzeid, A New Approach for Water Allocation Scheduling in Irrigation Open Channels......

\begin{tabular}{|c|l|c|c|c|c|c|c|c|}
\hline & Alawamer & & & & & & & \\
\hline 6 & $\begin{array}{l}\text { Northen } \\
\text { Alawamer }\end{array}$ & 780 & 475.02 & 232.44 & 72.54 & 408.72 & 256.62 & 113.88 \\
\hline 7 & Der Shew & 400 & 243.6 & 119.2 & 37.2 & 209.6 & 131.6 & 58.4 \\
\hline 8 & $\begin{array}{l}\text { Eastern Al } \\
\text { kadadeeh }\end{array}$ & 400 & 243.6 & 119.2 & 37.2 & 209.6 & 131.6 & 58.4 \\
\hline 9 & Al Hager & 500 & 304.5 & 149 & 46.5 & 262 & 164.5 & 73 \\
\hline 10 & Bani Ibraheem & 800 & 487.2 & 238.4 & 74.4 & 419.2 & 263.2 & 116.8 \\
\hline $10--1$ & $\begin{array}{l}\text { Bani Ibraheem } \\
\text { branch }\end{array}$ & 400 & 243.6 & 119.2 & 37.2 & 209.6 & 131.6 & 58.4 \\
\hline 11 & $\begin{array}{l}\text { Southern Al } \\
\text { Gabrawe }\end{array}$ & 500 & 304.5 & 149 & 46.5 & 262 & 164.5 & 73 \\
\hline 12 & $\begin{array}{l}\text { Northen Al } \\
\text { Gabrawe }\end{array}$ & 400 & 243.6 & 119.2 & 37.2 & 209.6 & 131.6 & 58.4 \\
\hline 13 & Alswalem & 700 & 426.3 & 208.6 & 65.1 & 366.8 & 230.3 & 102.2 \\
\hline 14 & Alam Aldeen & 500 & 304.5 & 149 & 46.5 & 262 & 164.5 & 73 \\
\hline 15 & Al Haraga & 500 & 304.5 & 149 & 46.5 & 262 & 164.5 & 73 \\
\hline
\end{tabular}

\subsection{Crop water requirements}

CROPWATER software was used for estimating reference evapotranspiration (ETo (mm/day)) by using the available meteorological data at Arab ALawamer local weather station as shown in Tables (3 and 4), as shown in Fig. (3) [25]. This program based on the Penman-Monteith method.

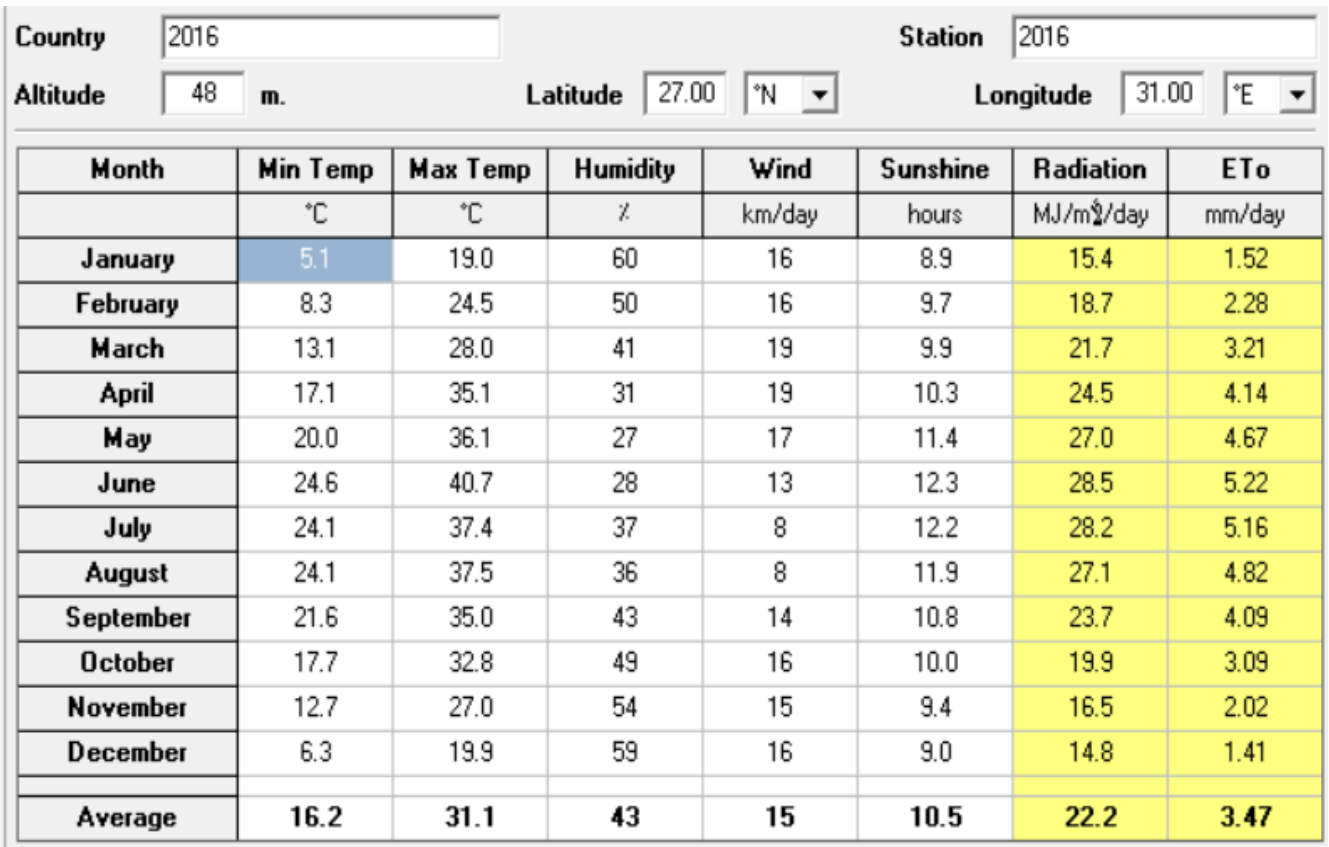

Fig. 3. Computer windows of CROPWATER software program. 


\subsection{Actual crop evapotranspiration}

Evapotranspiration from a cropped field is composed of transpiration from the crop and evaporation from the soil. The rate of evapotranspiration from the crop $\left(E T_{c}\right)$ or crop water use depends on the type of crop, stage of growth, moisture content of the surface soil, and the amount of energy available to evaporate water. Crop water use $\left(\mathrm{ET}_{\mathrm{c}}\right)$ is computed using the reference crop evapotranspiration $\left(\mathrm{ET}_{\mathrm{o}}\right)$ and a crop coefficient $\left(\mathrm{K}_{\mathrm{a}}\right)$ as follow:

$$
E T_{c}=K_{a} \times E T_{o}
$$

The crop coefficient $\left(\mathrm{K}_{\mathrm{a}}\right)$ depends on the growth and development of the crop canopy. It must include the basal crop coefficient $\left(\mathrm{K}_{\mathrm{Cb}}\right)$ and the effect of wet soil evaporation $\left(\mathrm{K}_{\mathrm{w}}\right)$. If water stress is expected, an appropriate stress factor $\left(\mathrm{K}_{\mathrm{s}}\right)$ can also be selected although this is generally not carried out [26 \& 27]. The average crop coefficient $\left(K_{a}\right)$ is estimated as:

$$
K_{a}=\left(K_{a b} \times K_{s}\right)+K_{w}
$$

$\mathrm{K}_{\mathrm{w}}=$ factor to account for increase evaporation (wet soil evaporation factor) [29].

$$
K_{w}=F_{w} \times\left(1-K_{c b}\right) \times A_{f}
$$

where $F_{w}=$ the fraction of the soil surface wetted that take 1.0 for basin irrigation and $A_{f}=$ the average wet soil evaporation factor as reported by Paul et al. [27]. The crop coefficient system developed by Doorenbos and Pruitt [30] and modified by Howell et al. [31] to estimate actual crop evapotranspiration. To use this method, the growing season is divided into four stages, as shown in Fig. (4):

Initial: Period from planting through early growth when the soil is not, or is hardly, covered by the crop (ground cover $<10 \%$ ).

Canopy development: Period from initial stage to the time that the crop effectively covers the soil surface (ground cover about 70 to $80 \%$ ).

Mid-season: Period from full cover until the start of maturation when leaves begin to change color or senesce.

Maturation: Period from end of mid-season until physiological maturity or harvest.

The progression of the basal crop coefficient during the season is illustrated in Fig. (4) for Yellow corn as an example. 


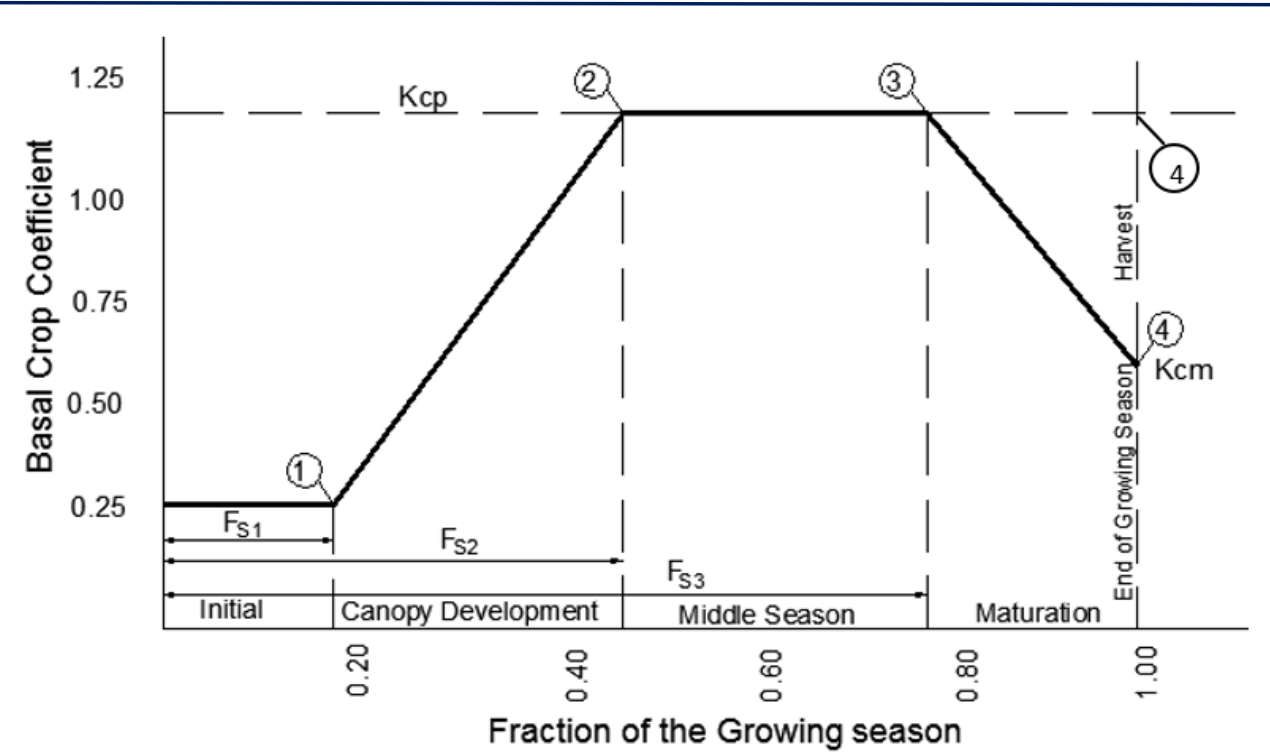

Fig. 4. Basal crop coefficient $\left(\mathrm{K}_{\mathrm{cb}}\right)$, [27].

To compute the crop coefficient during other periods of crop development, four points on the crop coefficient curve need to be defined. The first point is the fraction of the growing season where canopy development begins (point 1 in Fig. 4). At this point, the value of $K_{c b}(0.25)$ is known based on the assumption in the preceding paragraph, so only $\mathrm{F}_{\mathrm{S} 1}$ is needed. The second point occurs when the canopy has developed adequately to provide effective cover. Currently, the basal crop coefficient reaches its peak value. Thus, for the second point (point 2 in Fig. 4), both the peak values of $\mathrm{K}_{\mathrm{cb}}\left(\mathrm{K}_{\mathrm{cp}}\right)$ and $\mathrm{F}_{\mathrm{S} 2}$ are needed. Point 3 in Fig. 4 is the time when the crop begins to mature. The only value needed for the third point is the time $\left(\mathrm{F}_{\mathrm{S} 3}\right)$ because the crop coefficient at point 3 equals the peak value of the basal crop coefficient. For the fourth point, two locations are shown in Fig. (4). The lower location represents crops that begin to senescence before harvest. To define this point, the value of the basal crop coefficient at maturity $\left(\mathrm{K}_{\mathrm{cm}}\right)$ must be known. If the crop is harvested before the plant begins to mature, the crop coefficient remains constant at the peak value until harvest (the second location of point 4 in Fig. 4). Hence, the needed five definitions to compute the crop coefficient $\left(\mathrm{F}_{\mathrm{s} 1}, \mathrm{~F}_{\mathrm{S} 2}, \mathrm{~F}_{\mathrm{S} 3}, \mathrm{~K}_{\mathrm{cp}}, \mathrm{K}_{\mathrm{cm}}\right)$ are labeled in Fig. 4. Values for the five parameters needed to compute the basal crop coefficients for different crops in the study area are summarized in Table (7) [27]. The procedure to compute the basal_crop coefficient for any stage of growth is plotted in Fig. (5). Also, Table (8) shows the calculated values of the monthly average crop coefficient $\left(K_{a}\right)$ for the different crops in the study area. 
Table 7. Values of $\left(\mathrm{F}_{\mathrm{S} 1}, \mathrm{~F}_{\mathrm{S} 2}, \mathrm{~F}_{\mathrm{S} 3}, \mathrm{~K}_{\mathrm{cp}}, \mathrm{K}_{\mathrm{cm}}\right)$ for different crops, [27]

\begin{tabular}{|c|c|c|c|c|c|}
\hline Crop & $\mathrm{F}_{\mathrm{S} 1}$ & $\mathrm{~F}_{\mathrm{S} 2}$ & $\mathrm{~F}_{\mathrm{S} 3}$ & $\mathrm{~K}_{\mathrm{cp}}$ & $\mathrm{K}_{\mathrm{cm}}$ \\
\hline Wheat & 0.13 & 0.33 & 0.75 & 1.05 & 0.25 \\
\hline Bean & 0.16 & 0.42 & 0.80 & 1.15 & 0.25 \\
\hline Clover & 0.20 & 0.40 & 0.90 & 1.10 & 1.0 \\
\hline Yellow corn & 0.17 & 0.45 & 0.78 & 1.15 & 0.60 \\
\hline Sorghum & 0.16 & 0.42 & 0.75 & 1.10 & 0.55 \\
\hline Basil & 0.86 & 1 & 1 & 0.07 & 0.93 \\
\hline
\end{tabular}

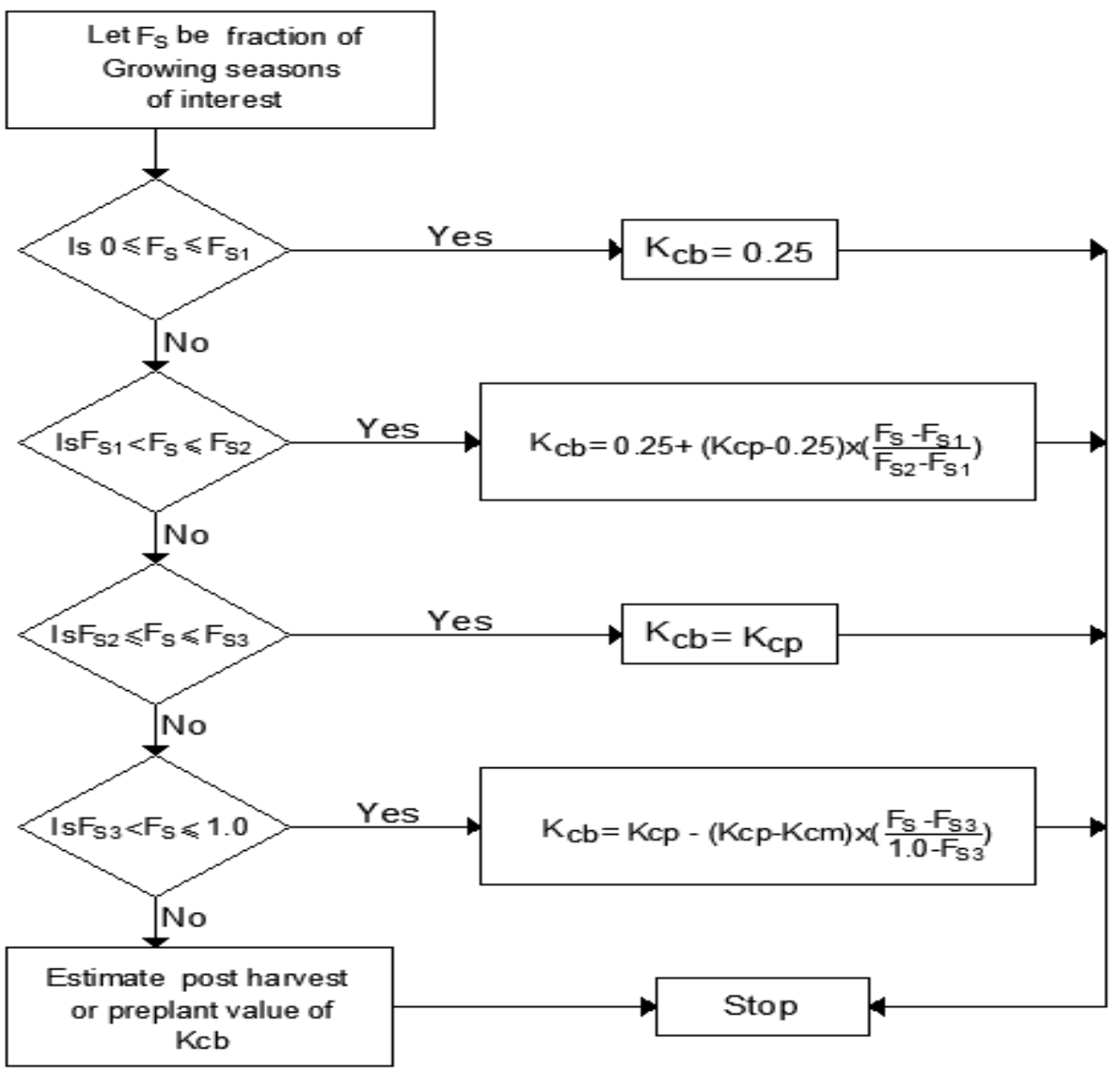

Fig. 5. Process to compute basal crop coefficient $\left(\mathrm{K}_{\mathrm{cb}}\right)$, [27]. 
Tarek S, Abuzeid, A New Approach for Water Allocation Scheduling in Irrigation Open Channels......

Table 8. Values of the monthly average crop coefficient $\left(K_{a}\right)$ for different crops.

\begin{tabular}{|c|c|c|c|c|c|c|c|c|c|c|c|}
\hline Crop & Month & $\begin{array}{l}\text { Days } \\
\text { of } \\
\text { month }\end{array}$ & $\begin{array}{c}\text { Day of } \\
\text { growing }\end{array}$ & $\begin{array}{c}\text { Time } \\
\text { since } \\
\text { planting }\end{array}$ & $\mathrm{F}_{\mathrm{s}}$ & $\mathrm{K}_{\mathrm{cb}}$ & $\mathrm{K}_{\mathrm{s}}$ & $\mathrm{F}_{\mathrm{w}}$ & $A_{f}$ & $\mathrm{~K}_{\mathrm{w}}$ & $\mathrm{K}_{\mathrm{a}}$ \\
\hline \multirow{6}{*}{$\sum_{\vec{J}}^{\vec{J}}$} & November & 30 & 15 & 15 & 0.09 & 0.25 & 1 & 1 & 0.511 & 0.38 & 0.63 \\
\hline & December & 31 & 31 & 46 & 0.28 & 0.84 & 1 & 1 & 0.511 & 0.08 & 0.92 \\
\hline & January & 31 & 31 & 77 & 0.46 & 1.05 & 1 & 1 & 0.511 & & 1.05 \\
\hline & February & 28 & 28 & 105 & 0.63 & 1.05 & 1 & 1 & 0.511 & & 1.05 \\
\hline & March & 31 & 31 & 136 & 0.82 & 0.83 & 1 & 1 & 0.511 & 0.09 & 0.92 \\
\hline & April & 30 & 30 & 166 & 1.00 & 0.25 & 1 & 1 & 0.511 & 0.38 & 0.63 \\
\hline \multirow{6}{*}{ ఐొ } & October & 31 & 15 & 15 & 0.10 & 0.25 & 1 & 1 & 0.511 & 0.38 & 0.63 \\
\hline & November & 30 & 30 & 45 & 0.30 & 0.73 & 1 & 1 & 0.511 & 0.14 & 0.87 \\
\hline & December & 31 & 31 & 76 & 0.51 & 1.15 & 1 & 1 & 0.511 & & 1.15 \\
\hline & January & 31 & 31 & 107 & 0.71 & 1.15 & 1 & 1 & 0.511 & & 1.15 \\
\hline & February & 28 & 28 & 135 & 0.90 & 0.7 & 1 & 1 & 0.511 & 0.15 & 0.85 \\
\hline & March & 31 & 15 & 150 & 1.00 & 0.25 & 1 & 1 & 0.511 & 0.38 & 0.63 \\
\hline \multirow{8}{*}{$\frac{\dot{亠}}{\grave{0}}$} & October & 31 & 15 & 15 & 0.07 & 0.25 & 1 & 1 & 0.511 & 0.38 & 0.63 \\
\hline & November & 30 & 30 & 45 & 0.21 & 0.45 & 1 & 1 & 0.511 & 0.28 & 0.73 \\
\hline & December & 31 & 31 & 76 & 0.35 & 0.94 & 1 & 1 & 0.511 & 0.03 & 0.97 \\
\hline & January & 31 & 31 & 107 & 0.50 & 1.1 & 1 & 1 & 0.511 & & 1.10 \\
\hline & February & 28 & 28 & 135 & 0.63 & 1.1 & 1 & 1 & 0.511 & & 1.10 \\
\hline & March & 31 & 31 & 166 & 0.77 & 1.1 & 1 & 1 & 0.511 & & 1.10 \\
\hline & April & 30 & 30 & 196 & 0.91 & 1.08 & 1 & 1 & 0.511 & & 1.08 \\
\hline & May & 31 & 20 & 216 & 1.00 & 1 & 1 & 1 & 0.511 & 0.00 & 1.00 \\
\hline \multirow{5}{*}{ 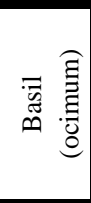 } & May & 31 & 15 & 15 & 0.11 & 0.25 & 1 & 1 & 0.511 & 0.38 & 0.63 \\
\hline & June & 30 & 30 & 45 & 0.33 & 0.86 & 1 & 1 & 0.511 & 0.07 & 0.93 \\
\hline & July & 31 & 31 & 76 & 0.55 & 1.15 & 1 & 1 & 0.511 & & 1.15 \\
\hline & August & 31 & 31 & 107 & 0.78 & 1.15 & 1 & 1 & 0.511 & & 1.15 \\
\hline & September & 30 & 30 & 137 & 1.00 & 1 & 1 & 1 & 0.511 & 0.00 & 1.00 \\
\hline \multirow{5}{*}{$\begin{array}{l}\Xi \\
0 \\
3 \\
0 \\
0 \\
0\end{array}$} & May & 31 & 15 & 15 & 0.10 & 0.25 & 1 & 1 & 0.511 & 0.38 & 0.63 \\
\hline & June & 30 & 30 & 45 & 0.30 & 0.76 & 1 & 1 & 0.511 & 0.12 & 0.88 \\
\hline & July & 31 & 31 & 76 & 0.51 & 1.15 & 1 & 1 & 0.511 & & 1.15 \\
\hline & August & 31 & 31 & 107 & 0.71 & 1.15 & 1 & 1 & 0.511 & & 1.15 \\
\hline & September & 28 & 28 & 135 & 0.90 & 0.6 & 1 & 1 & 0.511 & 0.20 & 0.80 \\
\hline \multirow{5}{*}{$\begin{array}{l}\Xi \\
\Xi \\
\vdots \\
\vdots \\
\vdots \\
\infty\end{array}$} & May & 31 & 15 & 15 & 0.11 & 0.25 & 1 & 1 & 0.511 & 0.38 & 0.63 \\
\hline & June & 30 & 30 & 45 & 0.33 & 0.29 & 1 & 1 & 0.511 & 0.36 & 0.65 \\
\hline & July & 31 & 31 & 76 & 0.55 & 1.1 & 1 & 1 & 0.511 & & 1.10 \\
\hline & August & 31 & 31 & 107 & 0.78 & 1.1 & 1 & 1 & 0.511 & & 1.10 \\
\hline & September & 30 & 30 & 137 & 1.00 & 0.55 & 1 & 1 & 0.511 & 0.23 & 0.78 \\
\hline
\end{tabular}

\subsection{Canal discharge computation}

Water consumption $(W C)$ in $\left(\mathrm{m}^{3} / \mathrm{sec}\right)$ needed for the areas served by different distributary canal in the study area is computed using the approach introduced by El-Enany et al. [32] which uses the following Eqn.:

$$
W C=\left(\frac{E T_{0} K_{a} A_{c i}}{1000}\right) \times \frac{4200}{86400}
$$


where $E T_{o}$ is the monthly average evapotranspiration for the area served by a distributary canal (mm/day), $K_{a}$ is monthly average crop coefficient, and $A_{\mathrm{ci}}$ is the area cultivated (feddan).

Table (9) shows the water consumption needed for the cultivated crops in the area served by Al Hammam distributary canal every month as an example. From this table, it is observed that, water consumption values of crops are different during the growth stages. As it is low at the beginning of cultivation (initial stage) and gradually increases to reach its highest value in stage of mid-season, then it returns to decline again in a maturation stage as shown previously in Fig. (4). Also, the last column in Table (9) shows the monthly total values of water consumption of crops grown in this area. It is noticed that the highest value of water consumption occurs in July, while the lowest value occurs in October, which is the beginning of the cultivation of winter crops and the end of the harvest of summer crops.

Hence, it turns out that large quantities of irrigation water can be provided when water is given to crops according to their water needs in the different growth stages and not at fixed values throughout the year. The discharges of the different distributary canals in the study area corresponding to the water consumption needed can be estimated by Eq. (6) [32].

$$
Q_{d}=W c \times \frac{N_{1}}{N_{2}} \times\left(\frac{24}{T_{B}-T_{g}}\right) \times\left(1+L_{R}\right) / \eta
$$

where $\mathrm{Q}_{\mathrm{d}}$ is the discharge of distributary canal during hours of irrigation $\left(\mathrm{m}^{3} / \mathrm{sec}.\right), \mathrm{T}_{\mathrm{e}}$ is the assumed day hour at which irrigation ends, $\mathrm{T}_{\mathrm{s}}$ is the assumed day hour at which irrigation starts, $L_{R}$ is the leaching requirement, which is a ratio of water consumptive use (\%) $\mathrm{L}_{\mathrm{R}}=0.05$, and $\eta$ is the irrigation efficiency for distributary canal, where conveyance and on-farm efficiencies are included $(=0.71)$ [32].

The difference between design and new discharges were calculated at each month to estimate the amount of water saved. Table (10) shows the value of design and new discharges of Al Hammam distributary canal during different months, as an example. 
Tarek S, Abuzeid, A New Approach for Water Allocation Scheduling in Irrigation Open Channels......

Table 9. Water consumption for the area served by Al Hammam distributary canal during month in (WC) $\mathrm{m}^{3} / \mathrm{sec}$, as an example

\begin{tabular}{|c|c|c|c|c|c|c|c|c|c|c|c|c|c|c|c|c|c|c|c|c|c|c|c|c|c|}
\hline \multirow{2}{*}{$\frac{\text { Crop }}{\sum_{\sum}^{\underline{\Xi}}}$} & \multicolumn{4}{|c|}{ Wheat } & \multicolumn{4}{|c|}{ Bean } & \multicolumn{4}{|c|}{ Clover (Berseem) } & \multicolumn{4}{|c|}{ Basil } & \multicolumn{4}{|c|}{ Yellow corn } & \multicolumn{4}{|c|}{ Surghum } & \multirow{2}{*}{ 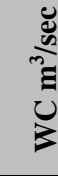 } \\
\hline & 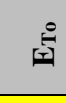 & 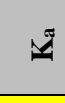 & $\bar{i}$ & $\bar{z}^{\bar{U}}$ & 도 & 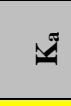 & $\bar{e}^{\overline{4}}$ & $\tilde{z}$ & $\stackrel{2}{2}$ & 气 & $\overline{4}$ & $3^{3}$ & $\stackrel{2}{\mathrm{I}}$ & تص & $\overline{4}$ & $\sum^{J}$ & $\stackrel{8}{\underline{1}}$ & ש & $\overline{4}$ & $z^{n}$ & $\stackrel{2}{\text { 되 }}$ & $\mathscr{1}$ & $\dot{e}^{-j}$ & $z^{8}$ & \\
\hline Jan & 1.64 & 1.05 & 2070.6 & 0.17 & 1.64 & 1.15 & 316.2 & 0.03 & 1.64 & 1.10 & 1013.2 & 0.09 & & & & & & & & & & & & & 0.29 \\
\hline Feb & 2.44 & 1.05 & 2070.6 & 0.26 & 2.44 & 0.85 & 316.2 & 0.03 & 2.44 & 1.10 & 1013.2 & 0.13 & & & & & & & & & & & & & 0.42 \\
\hline Mar & 3.35 & 0.92 & 2070.6 & 0.31 & 3.35 & 0.63 & 316.2 & 0.03 & 3.35 & 1.10 & 1013.2 & 0.18 & & & & & & & & & & & & & 0.52 \\
\hline Apr & 4.26 & 0.63 & 2070.6 & 0.27 & & & & & 4.26 & 1.08 & 1013.2 & 0.23 & & & & & & & & & & & & & 0.50 \\
\hline May & & & & & & & & & 5.07 & 1.00 & 1013.2 & 0.25 & 5.07 & 0.25 & 496.4 & 0.03 & 5.07 & 0.63 & 1781.6 & 0.28 & 5.07 & 0.63 & 1118.6 & 0.17 & 0.73 \\
\hline Jun & & & & & & & & & & & & & 5.66 & 0.86 & 496.4 & 0.12 & 5.66 & 0.88 & 1781.6 & 0.43 & 5.66 & 0.65 & 1118.6 & 0.2 & 0.75 \\
\hline Jul & & & & & & & & & & & & & 5.59 & 1.15 & 496.4 & 0.16 & 5.59 & 1.15 & 1781.6 & 0.56 & 5.59 & 1.10 & 1118.6 & 0.33 & 1.05 \\
\hline Aug & & & & & & & & & & & & & 5.28 & 1.15 & 496.0 & 0.15 & 5.28 & 1.15 & 1781.6 & 0.53 & 5.28 & 1.10 & 1118.6 & 0.32 & 0.99 \\
\hline Sep & & & & & & & & & & & & & 4.52 & 1 & 496.4 & 0.11 & 4.52 & 0.80 & 1781.6 & 0.31 & 4.52 & 0.78 & 1118.6 & 0.19 & 0.62 \\
\hline Oct & & & & & 3.30 & 0.63 & 316.2 & 0.03 & 3.30 & 0.63 & 1013.2 & 0.10 & & & & & & & & & & & & & 0.14 \\
\hline Nov & 2.03 & 0.63 & 2070.6 & 0.13 & 2.03 & 0.87 & 316.2 & 0.03 & 2.03 & 0.73 & 1013.2 & 0.07 & & & & & & & & & & & & & 0.23 \\
\hline Dec & 1.57 & 0.92 & 2070.6 & 0.15 & 1.57 & 1.15 & 316.2 & 0.03 & 1.57 & 0.97 & 1013.2 & 0.08 & & & & & & & & & & & & & 0.25 \\
\hline
\end{tabular}


Table 10. Values of designed, new, and saved discharge Al Hammam distributary canal during month in $(\mathrm{Q}) \mathrm{m}^{3} / \mathrm{sec}$, as an example

\begin{tabular}{|c|c|c|c|c|c|c|c|c|c|c|}
\hline Month & $\begin{array}{c}\text { Needed } \\
\text { Discharge } \\
\left(\mathrm{Q}_{c}\right)\end{array}$ & $\begin{array}{l}\text { Bed } \\
\text { width } \\
\text { (B) }\end{array}$ & $\begin{array}{c}\text { Needed } \\
\text { water } \\
\text { depth }(Y)\end{array}$ & $\begin{array}{l}\text { Water } \\
\text { slope } \\
(\mathrm{S})\end{array}$ & $\begin{array}{l}\text { Side } \\
\text { slope } \\
(\mathrm{Z})\end{array}$ & $1 / \mathrm{n}$ & $\begin{array}{l}\text { The lowest } \\
\text { water depth } \\
\text { that can be } \\
\text { used (Y) }\end{array}$ & $\begin{array}{c}\text { The discharge } \\
\text { corresponding } \\
\text { to the lowest } \\
\text { water depth (Q) }\end{array}$ & $\begin{array}{c}\text { Designed } \\
\text { discharge } \\
\left(Q_{\text {des }}\right)\end{array}$ & $\begin{array}{c}\text { The value of saved } \\
\text { discharge }(\mathrm{Q})\end{array}$ \\
\hline January & 0.86 & 4 & 0.62 & 0.00005 & $1: 1$ & 67 & 0.90 & 1.61 & 4.39 & 2.78 \\
\hline February & 1.25 & 4 & 0.78 & 0.00005 & $1: 1$ & 67 & 0.90 & 1.61 & 4.39 & 2.78 \\
\hline March & 1.55 & 4 & 0.88 & 0.00005 & $1: 1$ & 67 & 0.90 & 1.61 & 4.39 & 2.78 \\
\hline April & 1.47 & 4 & 0.46 & 0.00005 & $1: 1$ & 67 & 0.90 & 1.61 & 4.39 & 2.78 \\
\hline May & 2.17 & 4 & 1.07 & 0.00005 & $1: 1$ & 67 & 1.07 & 2.17 & 4.39 & 2.22 \\
\hline May & 2.22 & 4 & 1.09 & 0.00005 & $1: 1$ & 67 & 1.09 & 2.22 & 4.39 & 2.17 \\
\hline June & 3.09 & 4 & 1.31 & 0.00005 & $1: 1$ & 67 & 1.31 & 3.09 & 4.39 & 1.30 \\
\hline July & 2.92 & 4 & 1.27 & 0.00005 & $1: 1$ & 67 & 1.27 & 2.92 & 4.39 & 1.47 \\
\hline August & 1.82 & 4 & 0.97 & 0.00005 & $1: 1$ & 67 & 0.97 & 1.83 & 4.39 & 2.56 \\
\hline October & 0.40 & 4 & 0.39 & 0.00005 & $1: 1$ & 67 & 0.90 & 1.61 & 4.39 & 2.78 \\
\hline November & 0.68 & 4 & 0.54 & 0.00005 & $1: 1$ & 67 & 0.90 & 1.61 & 4.39 & 2.78 \\
\hline December & 0.73 & 4 & 0.54 & 0.00005 & $1: 1$ & 67 & 0.90 & 1.63 & 5.39 & 2.78 \\
\hline
\end{tabular}




\section{Results and Discussion:}

The required monthly discharge was calculated for each canal in the study area according to the water needs of the crops cultivated in that month. Also, water depth corresponding to each monthly discharge was calculated using Manning equation, considering that the canal bed width, the side slopes, and the longitudinal slope as constants. As shown in Fig. (6), a minimum water depth of $0.90 \mathrm{~m}$ in canals has been suggested if the required depth is less than this value. This value was suggested for the water depth to be the lowest water height above the inlet pipe of any field by about $25 \mathrm{~cm}$ to ensure that the water reaches these fields in the required quantities.

Table (11) shows the values of the designed, new, and saving discharges in all Almanna distributary canals. From this table, it is clear that:

- In most canals, values of the new discharges varied between 32 and $67 \%$ of the designed discharges.

- For Der Shew, and Al Hager canals, the value of the required discharge equal to the designed discharge.

- The total value of discharge saved from Almanna distributary canals equals to $14.95 \mathrm{~m}^{3} / \mathrm{sec}$, representing $48.68 \%$ of all irrigation water given to Almanna distributary canals.

Table 11. Value of designed, new, and saved discharge of Almanna off-taking canals

\begin{tabular}{|l|l|c|c|c|c|c|}
\hline No & \multicolumn{1}{|c|}{ Canal } & $\begin{array}{c}\text { Area } \\
\text { Fedd })\end{array}$ & $\begin{array}{c}\text { Bed } \\
\text { Width }\end{array}$ & $\begin{array}{c}Q_{\text {Designed }} \\
\left(\mathrm{m}^{3} / \mathrm{sec}\right)\end{array}$ & $\begin{array}{c}\mathbf{Q}_{\text {new }} \\
\left(\mathbf{m}^{3} / \mathbf{s e c}\right)\end{array}$ & $\begin{array}{c}\mathbf{Q}_{\text {saved }} \\
\left(\mathbf{m}^{3} / \mathbf{s e c}\right)\end{array}$ \\
\hline 1 & R. Almanna s. canal & 1000 & 2.0 & 2.05 & $\mathbf{1 . 2 1}$ & $\mathbf{0 . 8 4}$ \\
\hline 2 & Al Hammam & 3400 & 4.0 & 4.39 & $\mathbf{1 . 6 1 : 3 . 0 9}$ & $\mathbf{1 . 3 0 : 2 . 7 8}$ \\
\hline $2-1$ & Arab meter branch & 600 & 2.0 & 1.45 & $\mathbf{0 . 8 5}$ & $\mathbf{0 . 6 0}$ \\
\hline $2-2$ & Al Atawla & 500 & 1.5 & 1.65 & $\mathbf{0 . 9 5}$ & $\mathbf{0 . 7 0}$ \\
\hline $2-3$ & Al Zafran & 400 & 1.5 & 1.34 & $\mathbf{0 . 7 0}$ & $\mathbf{0 . 6 4}$ \\
\hline $2-4$ & Al Taweel & 500 & 1.5 & 2.92 & $\mathbf{0 . 9 9}$ & $\mathbf{1 . 9 3}$ \\
\hline 3 & Shew & 450 & 1.0 & 1.26 & $\mathbf{0 . 8 8}$ & $\mathbf{0 . 3 8}$ \\
\hline 4 & Western Al Awamer & 200 & 1.0 & 1.38 & $\mathbf{0 . 6 2}$ & $\mathbf{0 . 7 6}$ \\
\hline 5 & Eastern Alawamer & 400 & 1.0 & 0.94 & $\mathbf{0 . 6 2}$ & $\mathbf{0 . 3 2}$ \\
\hline 6 & Northen Alawamer & 780 & 1.5 & 2.05 & $\mathbf{0 . 6 7}$ & $\mathbf{1 . 3 8}$ \\
\hline 7 & Der Shew & 400 & 1.0 & 0.50 & $\mathbf{0 . 5 0}$ & $\mathbf{0 . 0 0}$ \\
\hline 8 & Eastern Al kadadeeh & 400 & 1.0 & 0.74 & $\mathbf{0 . 5 0}$ & $\mathbf{0 . 2 4}$ \\
\hline 9 & Al Hager & 500 & 1.5 & 0.95 & $\mathbf{0 . 9 5}$ & $\mathbf{0 . 0 0}$ \\
\hline 10 & Bani Ibraheem & 800 & 2.0 & 1.74 & $\mathbf{0 . 8 5}$ & $\mathbf{0 . 8 9}$ \\
\hline $10-1$ & Bani Ibraheem bran. & 400 & 1.0 & 1.45 & $\mathbf{0 . 5 0}$ & $\mathbf{0 . 9 5}$ \\
\hline 11 & Southern Al Gabrawe & 500 & 1.0 & 2.01 & $\mathbf{0 . 7 0}$ & $\mathbf{1 . 3 1}$ \\
\hline 12 & No.n Al Gabrawe & 400 & 1.0 & 0.81 & $\mathbf{0 . 5 0}$ & $\mathbf{0 . 3 1}$ \\
\hline 13 & Alswalem & 700 & 1.5 & 1.93 & $\mathbf{0 . 9 5}$ & $\mathbf{0 . 9 8}$ \\
\hline 14 & Alam Aldeen & 500 & 1.0 & 0.90 & $\mathbf{0 . 5 0}$ & $\mathbf{0 . 4 0}$ \\
\hline 15 & Al Haraga & 500 & 1.0 & 1.15 & $\mathbf{0 . 7 0}$ & $\mathbf{0 . 4 5}$ \\
\hline
\end{tabular}




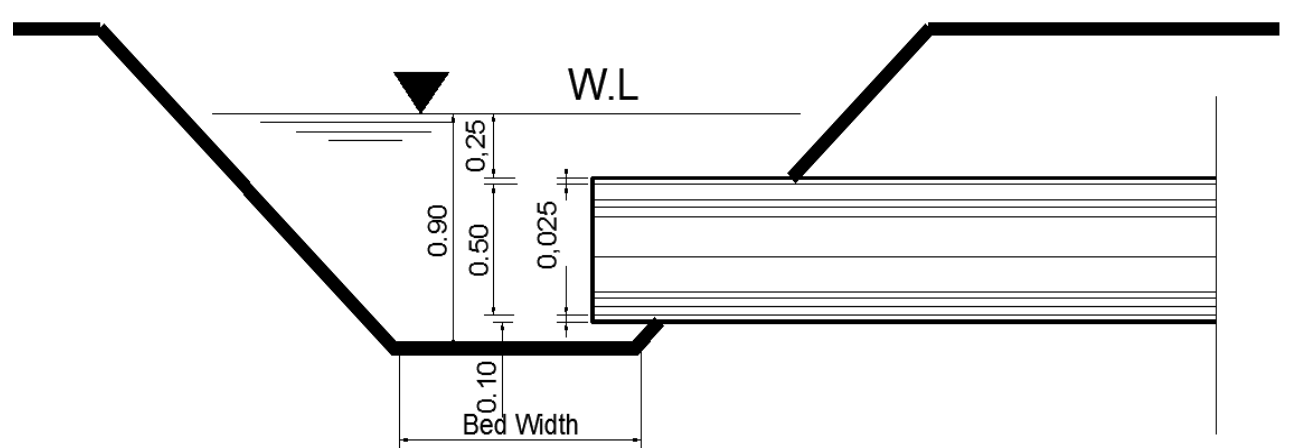

Fig. 6. Modified cross section of the canal

\section{Conclusions}

The study introduced and applied a new approach for water allocation scheduling in irrigation open channels. This approach is mainly depending on the good estimation of crop water needs in the each of plant growth stage. The main conclusions can be summarized as follows:

[1] Applying the introduced approach may lead to save a large amount of irrigation water.

[2] According to the total monthly values of water consumption of crops grown in the study area, the highest value of water consumption was in July, while the lowest value was in October, so the delivery flow rates should be scheduled accordingly.

[3] The percentage value of the new discharges of Almanna distributary canals, which calculated according to the water needs of crops cultivated in the area, varied between $32 \%$ and $67 \%$ of the design discharges.

[4] Applying the introduced approach on Almanna distributary canals can be saving a large amount of irrigation water that reaches about 19.375 Million cubic meters per month, which is representing $48.68 \%$ of all irrigation water given to Almanna distributary canals.

\section{References:}

[1] Chambers, R. (Ed.), (1988), "Managing Canal Irrigation: Practical Analysis from South Asia", Oxford Publishing, New Delhi, pp. 20:25.

[2] Levine, G., (1982). "Relative Water Supply: An Explanatory Variable for Irrigation Systems". Tech. Report No. (6). Cornell University, Ithaca, NY.

[3] Lenton, R.L., (1984). "A Note on Monitoring Productivity and Equity in Irrigation Systems. In: Pant, N. (Ed.), Productivity and Equity in Irrigation Systems". Ashish Publishing.

[4] Sampath, R.K., (1988). "Equity Measures for Irrigation System Performance Evaluation". Water Int. 13, 25 \pm 32.342 C. Santhi, N.V. Pundarikanthan / Agricultural Water Management 43 (2000) 327:343.

[5] Bos, M.G., and Nugteren, J., (1990). "On Irrigation Efficiencies". Publication 
Tarek S, Abuzeid, A New Approach for Water Allocation Scheduling in Irrigation Open Channels......

19. International Institute for Land Reclamation and Improvement, Wageningen.

[6] Molden, D.J., and Gates, T.K., (1990). "Performance Measures for Evaluation of Irrigation Water Delivery Systems". J. Irrigation Drainage Eng. ASCE 116(6), 804:823.

[7] Garces, C., (2000). "A Methodology to Evaluate the Performance of Irrigation Systems: application to Philippines National Systems”. Ph.D. Thesis. Cornell University, Ithaca, NY, unpubl.

[8] Smith, L.E.D., (1990). "An Economist Perspective on Irrigation Performance Assessment: With Examples from Large Scale Irrigation in Morocco". Irrigation Drainage Systems 4, 329:343.

[9] Abernethy, C.L., (1986). "Performance Measurement in Canal Water Management: A Discussion”. ODI/IIMI Irrigation Management Network. Paper No. 86/2d, pp. 1:10.

[10] Kalu, I.L., Paudyal, G.N., and Gupta, A.D., (1994). "Equity and efficiency issues in irrigation water distribution". Agricultural Water Management 28: $335-438$.

[11] Dudley, N.J., Howell, D.T., and Musgrave, W.F., (1971). "Optimal intraseasonal irrigation water allocation”. Water Resources Research 7(4): 770-788.

[12] Bras, R.L., and Cordova, J.R., (1981). "Intra-seasonal water allocation in deficit irrigation". Water Resources Research 17(4): 866-874.

[13] Loucks, D.P., Stedinger, J.R., and Haith, D.A., (1981). "Water Resources Systems Planning and Management". Prentice-Hall: Englewood Cliffs, NJ.

[14] Rajput, T.B.S., and Michael, A.M., (1989). "Scheduling of Canal Deliveries. I. Development of an Integrated Canal Scheduling Model". Irrigation and Power 46(2), 23:39.

[15] Vedula, S., Ramesh, T.S.V., and Mujumdar P.P. (1993). "Real-time Irrigation Scheduling". In International Conference on Environmentally Sound Water Resources Utilisation, Bangkok, Thailand, November; 25-31.

[16] Kalu, I.L., Paudyal, G.N., and Gupta, A.D., (1994). "Equity and efficiency issues in irrigation water distribution". Agricultural Water Management 28: $335-438$.

[17] Kan, C.E. and Shu, C.M., (1984). "Study in Drought Tolerance of Paddy Rice and Water Conservation for Irrigation". Journal of Taiwan Water Conservancy, 32 (1): 4-47 (Chinese).

[18] Khan, S., Tariq, R., Yuanlai, C., and Blackwell, J., (2006). "Can irrigation be sustainable?" Agricultural Water Management 80(1-3): 87-99.

[19] Santhi, C. and Pundarikanthan, N.V., (2000). "A new planning model for canal scheduling of rotational irrigation". Agricultural Water Management, 43: 327343.

[20] Yuanhua, Hongyuan, (1994). "Real-Time Operation Scheduling of Canal System with Rotational Irrigation”. International Conference on Irrigation Management Transfer, Wuhan, China.

[21] Hill, R.W., and Allen, R.G., (1996). "Simple Irrigation Scheduling Calendars". J. Irrigation Drainage Eng. ASCE, March/ April, pp. 107:111.

[22] Zhi, W., Mohan Reddy, J., Feyen, J., (1995). "Improved 0 \pm 1 Programming Model for Optimal Flow Scheduling in Irrigation Canals". Irrigation Drainage 
Systems 9, 105 \pm 116 .

[23] Wouter, W., Robert, S., Mohamed, N., Eman S. A., Jochen, F., and Henk, R., (2016). "Issues and Challenges in Spatial and Temporal Water Allocation in the Nile Delta". Sustainability, 8, 383.

[24] Arab Alawlamer Weather Station (2020), "Meteorological data of Abnoub cropped area during the period from 2014 to 2019 as documented in the Arab Alawlamer weather station", Assiut, Egypt.

[25] Allen, G.R., Pereira, L. S., Raes, D. and Smith, M., (1998). "Crop Evapotranspiration, Guidelines for Computing Crop Water Requirements" FAO. Irrigation and drainages paper No 56. Rome, Italy.

[26] Hanks, R.J., (1974). "Model for Predicting Plant Yield as Influenced by Water Use". Agronomy Journal, Vol.66, pp.660-665.

[27] Paul K. K., Gylan L. D., and Nelton O. S., (1993). "Part 623 National Engineering Handbook", Chapter 2, "Irrigation Water Requirements" Book, United States Department of Agriculture. Soil Conservation Service.

[28] Ritchie, J.T., (1973). "Influence of Soil Water Status and Meteorological Conditions on Evaporation from a Corn Canopy”. Agron. J. Vol. 65, No. E, pp. 893-897.

[29] Wright, J.L., (1981). "Crop Coefficients for Estimates of Daily Crop Evapotranspiration". In Proceedings of the irrigation scheduling conference: Irrig. sched. for water and energy conserv. In the 80's. Amer. Soc. Agric. Eng., St. Joseph, MI, pp. 18-26.

[30] Doorenbos, J., and Pruitt. W.O. (1977). "Guidelines for predicting crop water requirements". Irrig. and Drain. Paper No. 24, 2nd edition, Food and Agric. Organ. of the United Nations, Rome, Italy, $156 \mathrm{pp}$.

[31] Howell, T.A., Bucks, D.A., Goldhamer, D.A., and Lima, J. M. (1986). “Trickle irrigation for crop production", Develop. in Agric. Eng. 9. Elsevier, NY, pp. 241-279.

[32] El-Enany, M. A., El-Alfy, K. S., Sobeih, M. F., Armanious, S. D., and Gergis, E. S., (2004). "Modification of the Improved Irrigation System in the Old Lands in Egypt". Vol.29, No.1. 


\section{أسلوب جديا لجدولة توزيع المياه في قنوات الري المفتوحة حالة در اسةة: ترعة المعنا الرئيسية وفروعهاه}

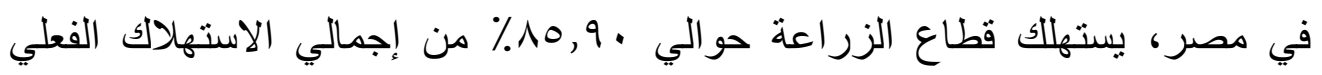

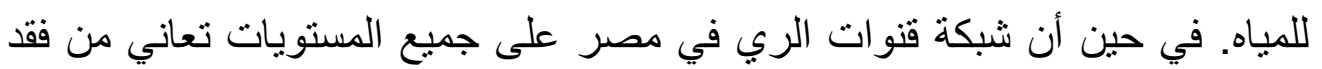

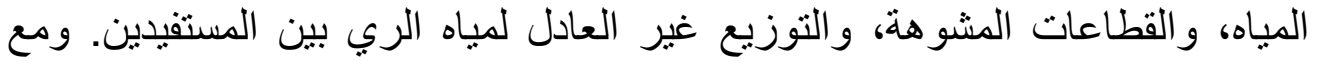
النمو السكاني والتوسع في الأنثطة الاقتصادية والصناعية ودخول مصر إلى مرحلة

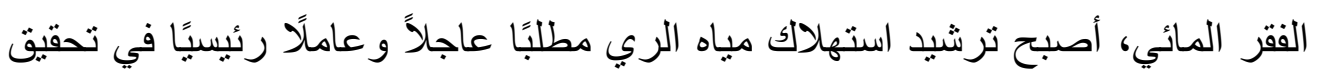

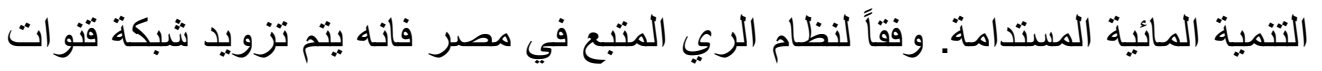

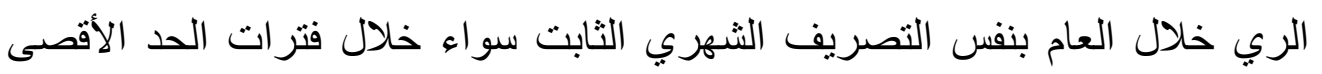

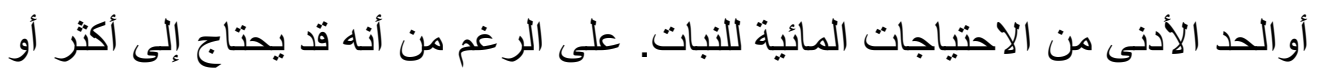
أقل من هذه الكميات خلال مراحل النمو المختلفة. الهدف من هذه الدراسة هو تفعيل أسلوب جديد لإدارة شبكة قنوات الري لمنطقة الدراسة. بسمح هذا الأسلوب بتزويد

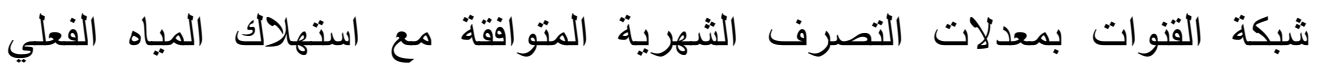
للمزروعات. وتساعد هذه الطريقة في ترشيد الاستهلاك خلال الموسم الزراعي. تم تطبيق الطريقة على قناة المعنا الرئيسية وفروعها التابعة لمحافظة أسيوط في وسط لهاه مصر، كمثال لنظام الري في مصر حيث يمارس التوزيع الدوراني على مستوى قناة

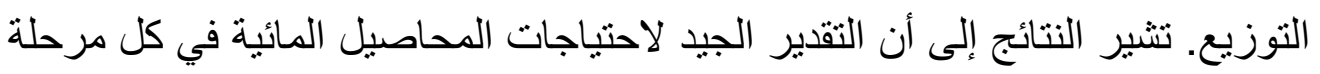

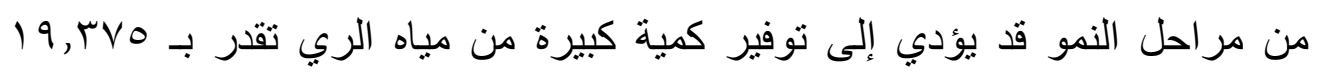

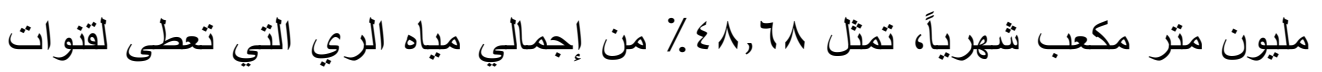

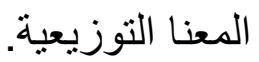

